ApJL 2010 in Press

\title{
The Genesis of an Impulsive Coronal Mass Ejection observed at Ultra-High Cadence by AIA on SDO
}

\author{
S. Patsourakos ${ }^{1}$, A. Vourlidas ${ }^{2}$, G. Stenborg ${ }^{3}$
}

\begin{abstract}
The study of fast, eruptive events in the low solar corona is one of the science objectives of the Atmospheric Imaging Assembly (AIA) imagers on the recently launched Solar Dynamics Observatory (SDO), which take full disk images in ten wavelengths with arcsecond resolution and 12 sec cadence. We study with AIA the formation of an impulsive coronal mass ejection (CME) which occurred on June 13, 2010 and was associated with an M1.0 class flare. Specifically, we analyze the formation of the CME EUV bubble and its initial dynamics and thermal evolution in the low corona using AIA images in three wavelengths (171, $193 \AA$, and $211 \AA$ ). We derive the first ultrahigh cadence measurements of the temporal evolution of the CME bubble aspect ratio (=bubble-height/bubble-radius). Our main result is that the CME formation undergoes three phases: it starts with a slow self-similar expansion followed by a fast but short-lived ( $\sim 70 \mathrm{sec}$ ) period of strong lateral over-expansion which essentially creates the CME. Then the CME undergoes another phase of self-similar expansion until it exits the AIA field of view. During the studied interval, the CME height-time profile shows a strong, short-lived, acceleration followed by deceleration. The lateral overexpansion phase coincides with the deceleration phase. The impulsive flare heating and CME acceleration are closely coupled. However, the lateral overexpansion of the CME occurs during the declining phase and is therefore not linked to flare reconnection. In addition, the multi-thermal analysis of the bubble does not show significant evidence of temperature change.
\end{abstract}

Subject headings: Sun: coronal mass ejections (CMEs)

\section{Introduction}

Synoptic observations of Coronal Mass Ejections (CMEs) have recorded thousands of events over the last 15 years leading to a decent understanding of their basic physical parameters (e.g.,

\footnotetext{
${ }^{1}$ University of Ioannina, Department of Physics, Section of Astrogeophysics, Ioannina, Greece

${ }^{2}$ Code 7663, Naval Research Laboratory, Washington, DC, USA

${ }^{3}$ Interferometrics, Inc, Herdon, VA, USA
} 
Schwenn et al. (2006)). However, their initiation and driving mechanisms remain unclear (Forbes et al. 2006) because their formation and initial stages of evolution occur in the low corona where, until recently, observational coverage was sparse and cadence was low.

Observationally, we can decompose the CME evolution into three stages: initiation, acceleration, and propagation (Zhang \& Dere 2006). Height-time measurements of CME fronts, using EUV imagers and white light coronagraphs, have shown a close correspondence between the initial CME acceleration phase and the impulsive phase of the soft X-ray (SXR) flare (Zhang et al. 2004; Temmer et al. 2008, 2010) and have established the CME acceleration profile as a proxy to the energy release. Detailed acceleration profiles, especially for impulsive CMEs, provide important constraints for all CME initiation models.

The understanding of CME formation is lacking behind the understanding of CME acceleration due to cadence and field of view restrictions. Most CME models agree that the final ejected structure is a magnetic fluxrope which may correspond to the cavity observed in 3-part CMEs in the outer corona (Subramanian \& Vourlidas 2007). But we do not yet know if the fluxrope preexists, if it forms on-the-fly or if the final structure is a combination of the above processes. The answer holds serious implications for the relative importance of ideal versus non-ideal mechanisms for the eruption process (e.g., Démoulin \& Aulanier 2010, and references therein). Impulsive CMEs are of particular interest because they tend to be associated both with strong flares and particle acceleration. However, the formation of impulsive CMEs is a particular challenge for existing instrumentation. They form low in the corona amidst significant pre-existing fine scale structure, they interact violently with their surroundings generating disturbances (such as waves and loop deflections that can obscure the evolution of the actual CME), and most of their energy release occurs within very short time intervals, at or below the typical cadence of most EUV or white light imagers.

Patsourakos et al. (2010) (hereafter, PVK10) analyzed relatively high cadence (75 - $150 \mathrm{sec})$ EUV STEREO observations of an impulsive CME. The CME had the appearance of a bubble and it was shown that the bubble was an early instance of the CME fluxrope seen higher up in the coronagraph field of view. The wide separation between the STEREO observations $\left(\sim 48^{\circ}\right)$ allowed PVK10 to perform detailed 3D modeling of the erupting bubble. The modeling showed that the bubble underwent a phase of lateral overexpansion (i.e., the bubble radius increasing faster than its height) very early in its evolution which coincided with the impulsive phase of the flare. The overexpansion transformed the bubble from a small, arcminute-scale feature into a large CMEscale fluxrope structure within 5 minutes. If this result holds in other events, it may explain the discrepancy between the sizes of CMEs and post-eruptive loops arcades and will clarify the true relationship between CMEs and their source regions. To accurately identify, delineate and measure the size of the erupting bubble, and the subsequent fluxrope, we need high cadence, high sensitivity off-limb EUV images which only the EUV imagers onboard the Solar Dynamics Observatory (SDO) can provide. In this Letter, we present the first multi-wavelength analysis of the early stages of a very impulsive CME with ultra-high cadence (12 sec) and compare the $3 \mathrm{D}$ evolution of the erupting 
bubble to the impulsive phase of the flare. Using three of the ten AIA channels sensitive to different temperatures we search for evidence of plasma heating. The observations and data analysis are presented in Section 2, and the results are discussed in Section 3.

\section{Observations and Data Analysis}

We use EUV imaging observations from AIA (AIA; Title et al. 2006) onboard the recently launched SDO and the Extreme Ultraviolet Imager (EUVI; Wuelser et al. 2004) onboard the Solar Terrestrial Relations Observatory (STEREO) Ahead spacecraft (STA). The eruption occurred on June 13, 2010 in active region NOAA 11079 which was located slightly behind the solar limb (S24W91). The eruption was accompanied by a rather short duration M1.0 flare starting at 05:33 UT, peaking at 05:39 UT and returning to pre-event levels at around 06:43 UT. Associated with this eruption was a small CME seen by the STEREO coronagraphs. STA was situated $74^{\circ}$ west from Earth, so AR11079 was $17^{\circ}$ east of STA's central meridian. The region was therefore observed in quadrature by the AIA and EUVI telescopes.

The eruption was observed as a limb event in all AIA channels with 12 sec cadence but here we use only the coronal channels centered at $171 \AA, 193 \AA$ and $211 \AA$ (Level 1.5 images) and the EUVI-A 195 Å channel (henceforth, we refer to a given channel simply by its central wavelength). The EUVI-A 195 cadence was 2.5 min.

The active region exhibited considerable activity throughout the day with loops rising and brightening but no major ejection was observed before our event. We focus on the eruption, and concentrate on the time interval from 05:30 to 05:43 UT. We provide two movies online in AIA 193 and EUVI-A 195 (movie1.mpg, movie2.mpg). Snapshots from these movies are shown in Figure 1. The image contrast is enhanced with a wavelet technique (Stenborg et al. 2008). Only the large scale brightness component has been removed.

The EUVI-A and AIA observations show the slow rise of loops over the active region for several minutes before the CME. Starting at $\sim$ 05:33 UT, brightenings appear very close to the surface at the inner core of the region. At the same time, a filament is clearly seen rising and heating (Figure 1. middle column). The filament seems to rotate as it rises which may indicate that it is kinking or writhing. The rise of the filament is associated with the rise of surrounding loops which eventually form an EUV cavity resembling a bubble around 05:36 UT. Taking advantage of the two viewpoints afforded by the EUVI-A and AIA observations we use triangulations to locate the erupting filament and the bubble in the two telescopes (see features marked in the righttmost column of Figure 1). 


\subsection{Formation of the bubble}

Thanks to the high cadence of the AIA images, we can study the formation and evolution of the EUV bubble in great detail. We provide online a base-ratio movie in 171 (movie3.mpg), where we divide each wavelet-enhanced image by a pre-event image at 05:30:23 UT. Representative snapshots from the movie are in Figure 2. The motion of the rising filament appears as twisting motion in AIA and straightening motion in EUVI-A. This pattern seems consistent with writhe

rather than kink (Török et al. 2010). As seen in AIA, the filament rises, twists and then starts falling towards the surface (after $\sim$ 05:33:11 UT). At that time, surrounding loops with seemingly different orientations begin to slowly grow (e.g., alternating black-white patterns in the lower two panels in Figure 21). Simultaneously, a ribbon-like intensity front propagates away from the filament along a south-east direction (upper two frames, Figure 2). The extent of this front roughly matches the lateral extension of the expanding loops. In the meantime, the expanding loops seem to pile up at the surface of the forming bubble (upper panel of Figure 21). The bubble becomes clear at $\sim$ 05:36 UT and continues to grow. After 2 minutes the bubble begins a strong lateral expansion which we analyze later. This time also signals the launch of an EUV wave around the bubble (movie1.mpg) but the wave analysis will be reported elsewhere. The outer rim of the bubble becomes progressively thinner and dimmer after 05:38:23 UT. After 05:42 UT, it increasingly becomes difficult to trace its upper part. The bubble exits the AIA field of view at $\sim 05: 45$ UT.

\subsection{Evolution of the bubble aspect ratio}

Following PVK10, we follow the evolution of the bubble aspect ratio to quantify the early 3D evolution of the CME. Unfortunately, the very impulsive nature of the event does not permit detailed two-viewpoint fitting of the bubble, as was done by PVK10. There is only one frame with the bubble fully formed in both EUVI-A 195 and AIA 193 (third column, Figure 1).

We are constrained to use the AIA data only. Our approach is to fit circles to the bubble rim using the 171 base-ratio images for the interval 05:35:23-05:42:23 UT. There are 36 images in all. We do not use other times because it was difficult to identify a significant fraction of the bubble rim with reasonable confidence due to either its slow evolution or faint signal. For each 171 base-ratio image, we manually select 10 points along the bubble rim and fit a circle to these points. We assume an error of 5 AIA pixels $(\sim 3$ arcsec $)$ in the determination of each point. This rather conservative estimate is the median of the half-width of the bubble rim for the 36 images. Figure 3 shows examples of the circle fits and demonstrates that a circle represents a good approximation to the upper section of the bubble. To further check the consistency of the circular assumption, we perform a circular fit to the EUVI-A bubble at 05:38:00 UT which yields a radius within 20\% of the AIA 193 fit at 05:38:06 UT (Figure 1). This agreement indicates that the bubble can be reasonably approximated by a $3 \mathrm{D}$ sphere.

The circular fits supply the radius and center height, relative to the solar surface, of the bubble. 
Hence the ratio between these two quantities provides the aspect ratio of the erupting bubble (Figure 4(a)). The error bars are determined by standard error propagation of the uncertainties of the height and radius measurements. If we assume that the bubble represents a rising fluxrope seen edge-on, we can interpret the derived aspect ratio as the ratio of the major to the minor axis of the erupting fluxrope. 1

The aspect ratio has a constant value of $\approx 1.7$ between $\sim$ 05:36:00 UT and $\sim$ 05:38:20 UT. The ratio decreases rapidly, within approximatively $70 \mathrm{sec}$, to a value of $\approx 1$ and remains relatively constant thereafter. The "jump" in the aspect ratio before $\sim$ 05:36 UT is possibly due to the uncertainty in the bubble determination at that time.

\subsection{Bubble acceleration profile and flare dynamics}

Following common practice, we measure the height-time $(H T)$ evolution of the EUV bubble in 171 by tracking a point in the bubble front between 05:34:00-05:43:11 UT. The resulting 47 measurements are shown in Figure 4(b). As done frequently, the HT data are first smoothed to reduce small-scale fluctuations (e.g., Vršnak et al. 2007). We use a smoothing cubic spline scheme which minimizes a function consisting of the sum of a $\chi^{2}$ fit of the data with a cubic spline plus a penalty function proportional to the second derivative of the cubic spline multiplied by a usersupplied factor $0 \leq$ spar $\leq 1$,e.g. Weisberg (2005). Smoothing factors between $0.7-0.8$ provide the best compromise between noisy (zero or small spar) and extremely smoothed (large spar) acceleration profiles. We adopt the median of the above interval (spar $=0.75)$ for the smoothing of the HT data (Figure $4(\mathrm{~b})$ ) which are then differentiated with respect to time to obtain velocity and acceleration profiles (panels (c) and (d) of Figure 4). The error bars are derived from the maximum residuals between the curves calculated with the median spar and those for spar 0.7 and 0.8. The errors in the speed and acceleration profiles resulting from the use of 5-pixel error estimate in the HT measurements were very small and were thus neglected. The results show that the bubble accelerates rapidly (within $4 \mathrm{~min}$ ) to a speed of $\approx 400 \mathrm{~km} \mathrm{~s}^{-1}$ and then starts to decelerate (Figure 4c). The acceleration profile is characterized by a short-lived $(F W H M \approx 2$ min) acceleration pulse followed by deceleration. The latter is expected to occur even in the inner corona, for impulsively accelerated CMEs (e.g., Temmer et al. (2010)). This may be due to solar wind drag as the CME speed exceeds the local solar wind speed. The deceleration is verified by the LASCO C2 measurements which show a speed of $\approx 200 \mathrm{~km} \mathrm{~s}^{-1}$.

The acceleration profile corresponds closely to the time derivative of the GOES soft X-ray (SXR) light curve, a proxy for hard X-rays (HXR) and hence for the flare reconnection2, (Figure 4(d)), as generally expected (e.g., Zhang et al. 2004; Temmer et al. 2008, 2010). The slower

\footnotetext{
${ }^{1}$ The spherical approximation applies only to the upper section of the flux-rope and not to its legs.

${ }^{2}$ RHESSI was observing the Crab Nebula during our event.
} 
SXR rise profile arises from the occultation of a significant part of the flaring region from Earth. The SXR loops contribute to the GOES lightcurve only after they rise above the solar limb. The SXR levels are high when the bubble forms ( 05:36 UT). Both the SXR derivative (and hence reconnection) and the bubble acceleration are close to zero when the strong lateral expansion starts. It is an indication that the expansion may be driven by mostly an ideal process. We will return to this important point in the Discussion.

\subsection{Multi-temperature behavior of the bubble}

We investigate the thermal behavior of the expanding bubble using the almost simultaneous AIA observations with filters sensitive to coronal plasmas of different temperatures. We use the observations in 171, 193, and 211 channels with peak responses at $0.8,1.25$, and $1.6 \mathrm{MK}$, respectively.

We posted an online movie of the 171/193 intensity ratio during the event (movie4.mpg). The observations between the two channels are separated by only $5 \mathrm{sec}$ which corresponds to a displacement of around 4 pixels at the maximum speed of $\approx 300 \mathrm{~km} \mathrm{~s}^{-1}$. This separation is smaller than the 10 pixel full width of the erupting rim and so it has minimal impact in the calculated intensity ratios. Snapshots from the intensity ratio movie are shown in Figure 5 , Brighter (darker) areas could signify relatively larger (lower) amounts of warmer (hotter) plasmas 3 . Such areas seem to remain unaltered which implies that the temperature does not vary substantially during the event. In Figure 5, the bright/dark segments of the bubble remain as such during its evolution.

To further quantify the thermal evolution of the bubble, we construct lightcurves by extracting the intensity along the propagation path between 05:30 UT and 05:42 UT in each channe 4 . The intensities at 5:30 UT are subtracted from each exposure. The peak intensity for the basesubtracted lightcurves is used as a proxy for the bubble intensity. All three lightcurves peak nearly simultaneously and at about the same time as the strong lateral expansion of the bubble (Figure 4). Also, the lightcurves have similar lifetimes to the acceleration pulse ( $F W H M \approx 2 \mathrm{~min})$. The 171 lightcurve, however, increases at a slower rate than either the 193 or 211 curves. This may be because the loops rising from the AR core were emitting mostly in 171 . The hotter emissions seem to lie around these 171 loops and the bubble in those channels seems to form as a result of the initial expansion of the 171 structures. The discussion above suggests that the bubble temperature did not vary substantialy during its expansion.

\footnotetext{
${ }^{3}$ Note that filter-ratios are not strictly monotonic functions of temperature over large temperature intervals.

${ }^{4}$ The intensities in the hotter channels, i.e. 335, 94, 131 were affected by diffraction patterns from the flare emission and were unsuitable for quantitative analysis.
} 


\section{Discussion and Conclusions}

Arguably, our most interesting finding is the strong lateral overexpansion of the bubble that in essence 'inflates' an initially small-scale feature into a CME-scale structure. This period of inflation starts after the main phase of flare heating and CME acceleration and argues strongly for ideal MHD rather than a reconnection-related process. The observed overexpansion is a considerable challenge for any CME model. The overexpansion may be triggered when the expanding CME 'exits' above the AR loop core and encounters weaker magnetic fields. To achieve pressure balance with its surroundings, the CME bubble must expand laterally (Moore et al. 2007). Another explanation may be flux conservation around a rising fluxrope of decreasing current (PVK10). However, the overexpansion in PVK10 was relatively well-synchronized with the impulsive flare phase and hence a reconnection-based driver was possible. The differences between these impulsive CME events indicate that the characteristics of lateral overexpansion in the early phase of CMEs vary from event to event making their study a potentially sensitive diagnostic of CME evolution and coronal properties. For instance, a deeper study of this event may supply estimates on the magnetic field strength of the regions in the vicinity of the eruption site. A detailed MHD modeling of the PKV10 event to determine the relative role of ideal vs non-ideal processes is underway.

Our multi-temperature observations show no appreciable temperature changes in the bubble during the event. If significant plasma heating (or cooling) was taking place, it would have led to temporally displaced lightcurves in the different channels (e.g., Robbrecht \& Wang 2010). This does not necessarily preclude heating (and then cooling) of the bubble plasma during its formation. According to standard flare-CME theory, field lines reconnecting in the vertical current sheet underneath the erupting flux become part of the CME bubble (e.g., Lin et al. 2004). If these lines fill with hot plasma (i.e., by evaporation) at a slower rate than the eruption, then no temperature changes may be detected. Indeed, for the analyzed AIA channels $(\approx 0.5-3 \mathrm{MK})$, the evaporation speeds are around $100 \mathrm{~km} \mathrm{~s}^{-1}$ (e.g., Milligan \& Dennis 2009) - slower than the CME $\operatorname{speed}\left(\sim 200-400 \mathrm{~km} \mathrm{~s}^{-1}\right)$. More analysis is needed to test these ideas further.

While reconnection may not be important in the lateral overexpansion of the bubble, it could be a significant factor for its formation. The bubble forms from a set of pre-existing loops at varying orientations during the main flare phase. This is consistent with the transformation of a loop arcade to a fluxrope structure and is predicted by several CME models (e.g., Lynch et al. 2008). However, the possible eruption trigger lies in the rise and possible instability of a small filament. Such structure could be related to a pre-existing fluxrope. At any rate, our observations of the initially very small bubble radius $\left(\sim 0.03 R_{\odot}\right)$ set an upper limit for the size of any pre-existing flux rope. We suggest that the observed lateral overexpansion may well the process through which eruptions starting small in the corona become large-scale CMEs further out. Analysis of more events is required to establish or refute this suggestion.

To summarize, we have presented EUV observations of the genesis of an impulsive CME. By taking full advantage of the unique SDO AIA capabilities - its unprecedented high cadence (12 
sec) and multi-temperature coverage - we were able to resolve the various stages of the event in great detail. Our main findings are:

- A set of slowly rising loops, possibly triggered by a rising and maybe kinking or writhing filament, is transformed into an EUV bubble within 2 minutes.

- The EUV bubble forms when both flare heating and CME acceleration are at their maximum levels.

- The bubble experiences a 70 sec period of strong lateral overexpansion followed by self-similar evolution.

- The lateral over-expansion starts when flare reconnection and CME acceleration are well through their peaks.

- The bubble rim emission shows no significant evidence of temperature change.

The AIA data used here are courtesy of SDO (NASA) and the AIA consortium. We thank the AIA team for the easy access to calibrated data and the referee for useful comments. The SECCHI data are courtesy of STEREO and the SECCHI consortium.

\section{REFERENCES}

Démoulin, P., \& Aulanier, G. 2010, ApJ, 718, 1388

Forbes, T. G., et al. 2006, Space Sci. Rev., 123, 251

Lin, J., Raymond, J. C., \& van Ballegooijen, A. A. 2004, The Astrophysical Journal, 602, 422435

Lynch, B. J., Antiochos, S. K., DeVore, C. R., Luhmann, J. G., \& Zurbuchen, T. H. 2008, ApJ, 683, 1192

Milligan, R. O., \& Dennis, B. R. 2009, ApJ, 699, 968

Moore, R. L., Sterling, A. C., \& Suess, S. T. 2007, ApJ, 668, 1221

Patsourakos, S., Vourlidas, A., \& Kliem, B. 2010, ArXiv e-prints

Robbrecht, E., \& Wang, Y.-M. 2010, The Astrophysical Journal, 720, L88

Schwenn, R., et al. 2006, Space Science Reviews, 123, 127

Stenborg, G., Vourlidas, A., \& Howard, R. A. 2008, ApJ, 674, 1201

Subramanian, P., \& Vourlidas, A. 2007, A\&A, 467, 685 
Temmer, M., Veronig, A. M., Kontar, E. P., Krucker, S., \& Vršnak, B. 2010, ApJ, 712, 1410

Temmer, M., Veronig, A. M., Vršnak, B., Rybák, J., Gömöry, P., Stoiser, S., \& Maričić, D. 2008, ApJ, 673, L95

Title, A. M., Hoeksema, J. T., Schrijver, C. J., \& The Aia Team. 2006, in COSPAR, Plenary Meeting, Vol. 36, 36th COSPAR Scientific Assembly, 2600-+

Török, T., Berger, M. A., \& Kliem, B. 2010, A\&A, 516, A49+

Vršnak, B., Maričić, D., Stanger, A. L., Veronig, A. M., Temmer, M., \& Roša, D. 2007, Sol. Phys., 241,85

Weisberg, S. 2005, Applied linear regression (John Wiley and Sons)

Wuelser, J., et al. 2004, in Society of Photo-Optical Instrumentation Engineers (SPIE) Conference Series, Vol. 5171, Society of Photo-Optical Instrumentation Engineers (SPIE) Conference Series, ed. S. Fineschi \& M. A. Gummin, 111-122

Zhang, J., \& Dere, K. P. 2006, ApJ, 649, 1100

Zhang, J., Dere, K. P., Howard, R. A., \& Vourlidas, A. 2004, ApJ, 604, 420

This preprint was prepared with the AAS $\mathrm{LAT}_{\mathrm{E}} \mathrm{X}$ macros v5.2. 


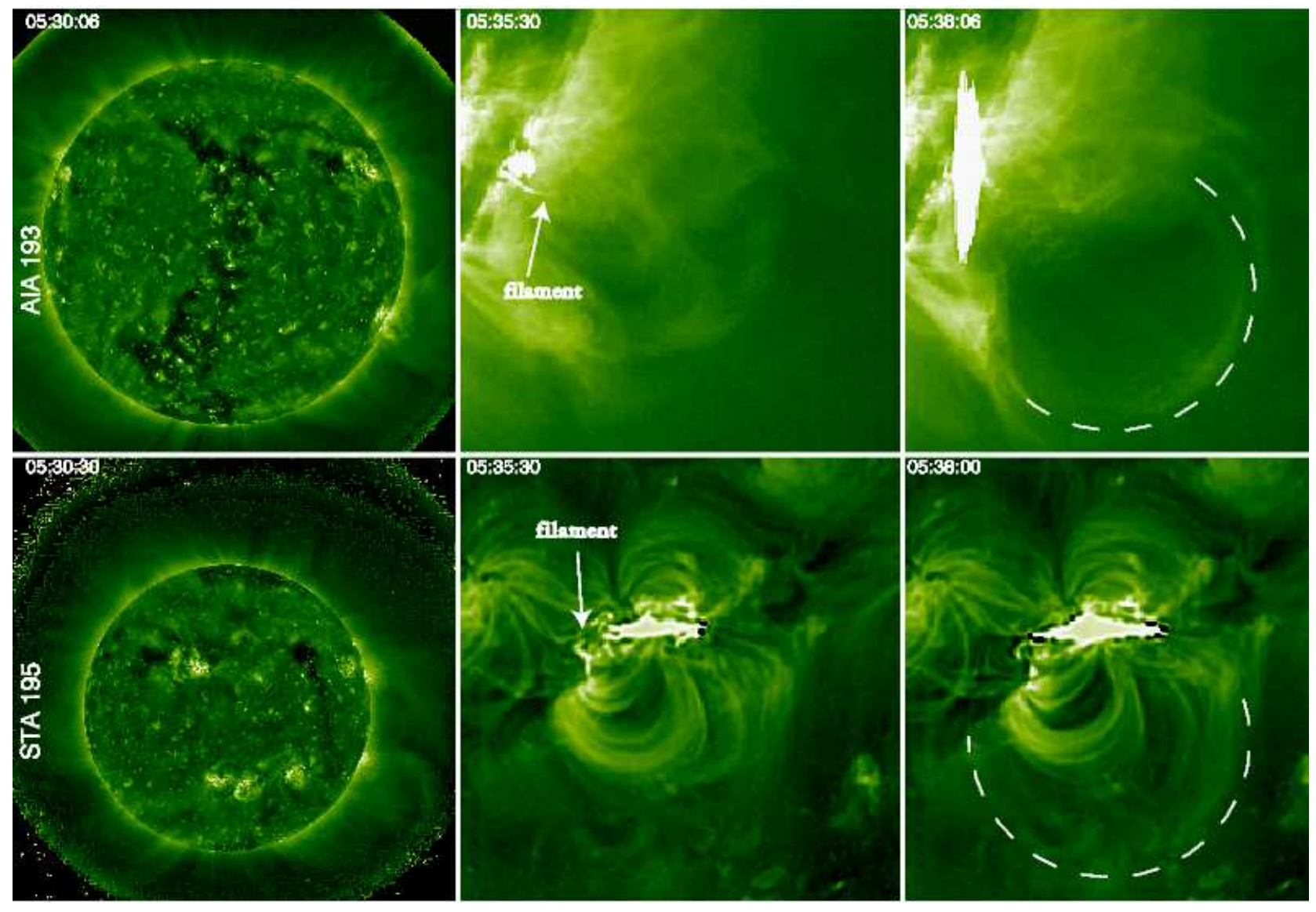

Fig. 1. - EUV Observations of the erupting active region in AIA 193 (upper panel) and the EUVIA 195 channel (lower panel). An untwisting filament apparently associated with the initiation of the eruption is marked by arrows. The dashed lines outline the expanding bubble. EUVI-A is situated $74^{\circ}$ West from Earth. 

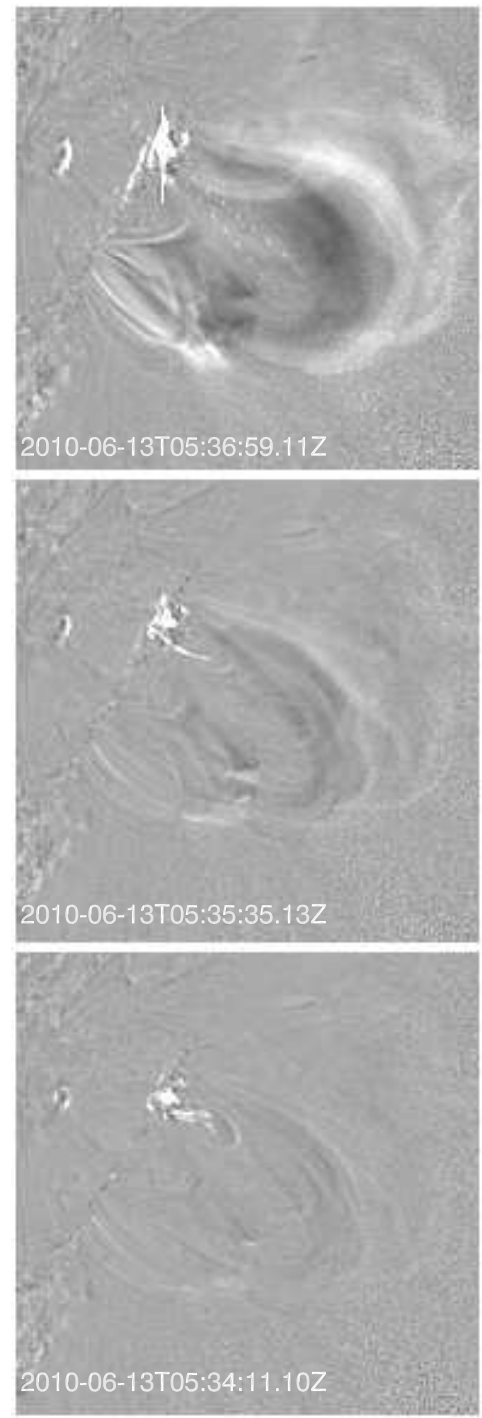

Fig. 2.- Representative AIA base-ratio 171 frames before and towards the EUV bubble formation. The ratio increases with from black to white within the range 0.3-1.4. 


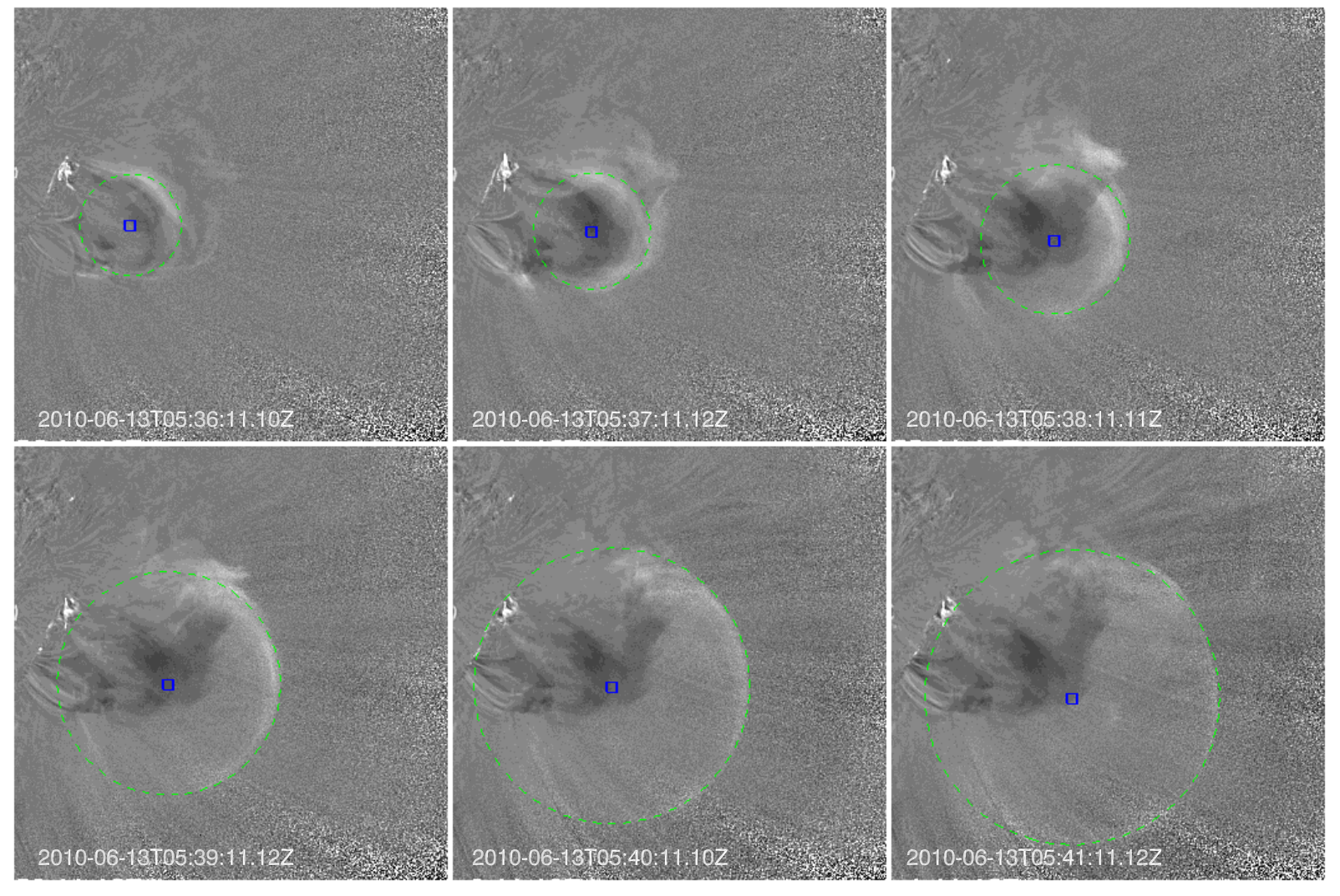

Fig. 3.- Representative circular fits (green lines) of the bubble in AIA 171 base-ratio images. The blue box marks the bubble center. The image at 05:30:23 UT is used as the base. 

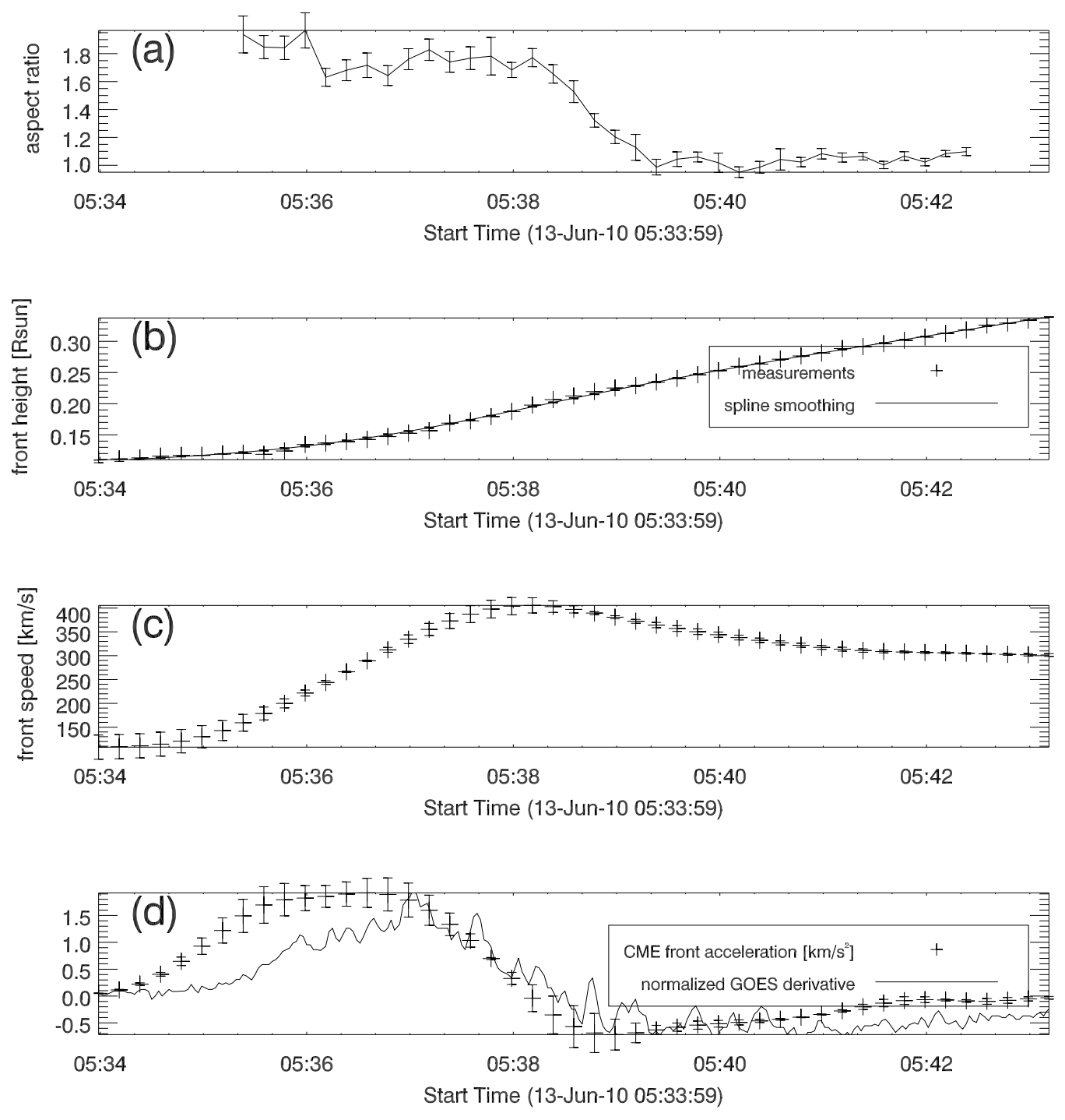

Fig. 4.- (a) Temporal evolution of the aspect ratio of the bubble. (b) HT measurements of the bubble front (crosses). The points are connected with spline smoothing curve (solid line). (c) Speed versus time curve prouced by differentiating the smoothed HT measurements. (d) Acceleration versus time curve (crosses) over-plotted on the time-derivative of the GOES SXR curve (solid line). 

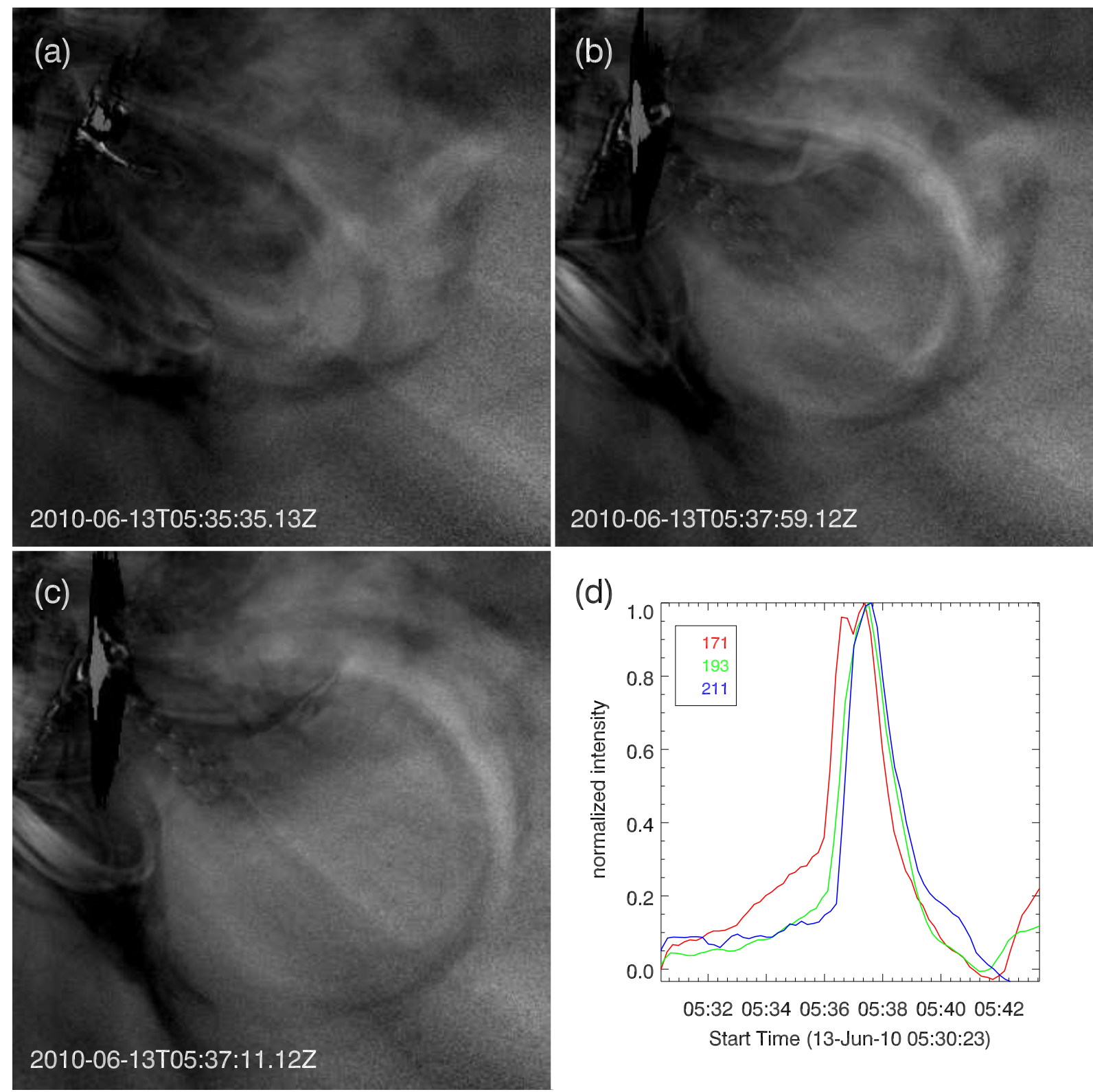

Fig. 5.- (a)-(c) AIA 171/193 intensity ratio snapshots. (d) Light-curves across the bubble rim in 171, 193, and 211 with pre-event intensity levels subtracted. The times on the images correspond to 171 and the 193 images trail by 5 sec. 